\title{
Genotypes and haplotypes of the VEGF gene are associated with higher mortality and lower VEGF plasma levels in patients with ARDS
}

\author{
R Zhai, M N Gong, W Zhou, T B Thompson, P Kraft, L Su, D C Christiani
}

Thorax 2007;62:718-722. doi: 10.1136/thx.2006.069393

See end of article for authors' affiliations

Correspondence to Dr David C Christiani, Harvard School of Public Health, 665 Huntington Avenue, Boston,

Massachusetts 02115 , USA; dchristi@hsph.harvard.edu

Received 27 July 2006 Accepted 9 January 2007

Published Online First

8 February 2007
Background: Endothelial injury is an important prognostic factor in acute respiratory distress syndrome (ARDS). Vascular endothelial growth factor (VEGF) plays a critical role in endothelial destruction and angiogenesis. Genetic variations of the VEGF gene have been associated with VEGF production. A study was undertaken to investigate the impact of VEGF gene polymorphisms on the clinical outcomes of ARDS.

Methods: Three VEGF polymorphisms $(-460 \mathrm{C} / T,+405 \mathrm{C} / \mathrm{G}$ and $+936 \mathrm{C} / T)$ were determined in 1253 patients in an intensive care unit with risk factors for ARDS, 394 of whom developed ARDS. Patients were followed for assessment of 60 day survival. Plasma VEGF levels were measured in 71 patients with ARDS. Results: The +936TT (OR 4.29, 95\% Cl 1.12 to 16.40, $\mathrm{p}=0.03$ ) and +936CT+TT (OR 1.98, 95\% Cl 1.14 to $3.42, p=0.01$ ) genotypes were significantly associated with increased mortality from ARDS. Plasma VEGF levels in patients with ARDS with the $+936 C T+\pi$ genotype were significantly lower than in subjects with the +936CC genotype (median 49 (IQR 16-98) pg/ml vs 112 (IQR 47-162) pg/ml, p=0.02). At the haplotype level, haplotype TCT $(-460 T+405 C+936 T)$ was significantly associated with a higher rate of mortality (OR 2.89, $95 \% \mathrm{Cl} 1.30$ to $6.43, p=0.009)$ and haplotype CGT $(-460 C+405 G+9367)$ was associated less strongly with increased mortality (OR $1.90,95 \% \mathrm{Cl} 0.94$ to $3.83, \mathrm{p}=0.07$ ) in patients with ARDS. Lower plasma VEGF levels were correlated with the probability of haplotype CGT (coefficient $=-0.26, p<0.05$ ), but the same trend of correlation was not significant to haplotype TCT.

Conclusions: VEGF polymorphisms may contribute to the prognosis and inter-individual variations in circulating VEGF levels in patients with ARDS.
A cute respiratory distress syndrome (ARDS) is lung injury characterised by alveolar injury and increased pulmonary vascular permeability. ${ }^{12}$ The pathogenetic basis of ARDS is incompletely understood; however, emerging evidence has suggested that the severity and outcome of ARDS depend significantly on the balance between alveolar epithelial and/or vascular endothelial injuries and their repair mechanisms. ${ }^{2-6}$

Vascular endothelial growth factor (VEGF) is an endothelial cell-specific mitogen that promotes angiogenesis and mediates vascular permeability. ${ }^{7}$ Overexpression of VEGF in the lungs induces increased pulmonary vascular permeability resulting in pulmonary oedema. ${ }^{8}$ However, VEGF expression in human lung epithelial cells can also increase neovascularisation, thereby contributing to the repair of endothelial injuries. ${ }^{69}$ In human lungs, low levels of VEGF were associated with the severity of ARDS while raised VEGF levels were associated with recovery from ARDS, indicating a role for VEGF in the repair process of lung injury. ${ }^{10-13}$

The VEGF gene is located on chromosome 6p31.3. Several functional single nucleotide polymorphisms (SNPs) in this gene have been described in the literature. The $+936 \mathrm{C} / \mathrm{T}$ polymorphism (rs3025039) has been related to lower levels of plasma VEGF, ${ }^{14} 15$ whereas the $-460 C / T$ (rs83306l) and $+405 C / G$ (rs2010963) polymorphisms have been shown to significantly increase VEGF production. ${ }^{16}$ Recent studies have indicated that these three SNPs are implicated in the risk of several disorders in which vascular injury and acceleration of inflammation are critical in disease development. ${ }^{18}$ However, the combined effect of these polymorphisms on the prognosis of ARDS has not been evaluated. Based on the biological function of these three VEGF polymorphisms on VEGF production and the pathological significance of VEGF in ARDS, we hypothesised that $V E G F$ polymorphisms may contribute to the poor outcomes in ARDS, and correlate with circulating VEGF levels in patients with ARDS.

\section{METHODS}

\section{Study subjects}

The present study was part of an ongoing research investigating the effects of multiple genetic variants on the risks or prognosis of ARDS. Recruitment of subjects was started in September 1999 and will continue until 2010. Details of the study have been described previously. ${ }^{19}{ }^{20}$ Briefly, all admissions to the intensive care unit (ICU) at Massachusetts General Hospital were screened daily for study-defined clinical risk factors for ARDS such as sepsis, trauma, aspiration or massive transfusions and were followed prospectively during their stay in the ICU for the development of ARDS (fig 1). Subjects were classified as having ARDS if they were intubated on positive ventilation and met the American European Consensus Committee (AECC) criteria for ARDS: (1) arterial oxygen tension/fraction of inspired oxygen ratio of $\leqslant 200 \mathrm{~mm} \mathrm{Hg}$; (2) bilateral infiltrates on chest radiographs; and (3) pulmonary arterial occlusion pressure $\leqslant 18 \mathrm{~mm} \mathrm{Hg}$ or no clinical evidence of left atrial hypertension. All cases of ARDS were followed for all-cause 60 day mortality. The study was approved by the Human Subjects Committees of Massachusetts General Hospital and the Harvard School of Public Health, Boston, and informed written consent was obtained from all subjects or their appropriate surrogates.

Abbreviations: ARDS, acute respiratory distress syndrome; SNP, single nucleotide polymorphism; VEGF, vascular endothelial growth factor 


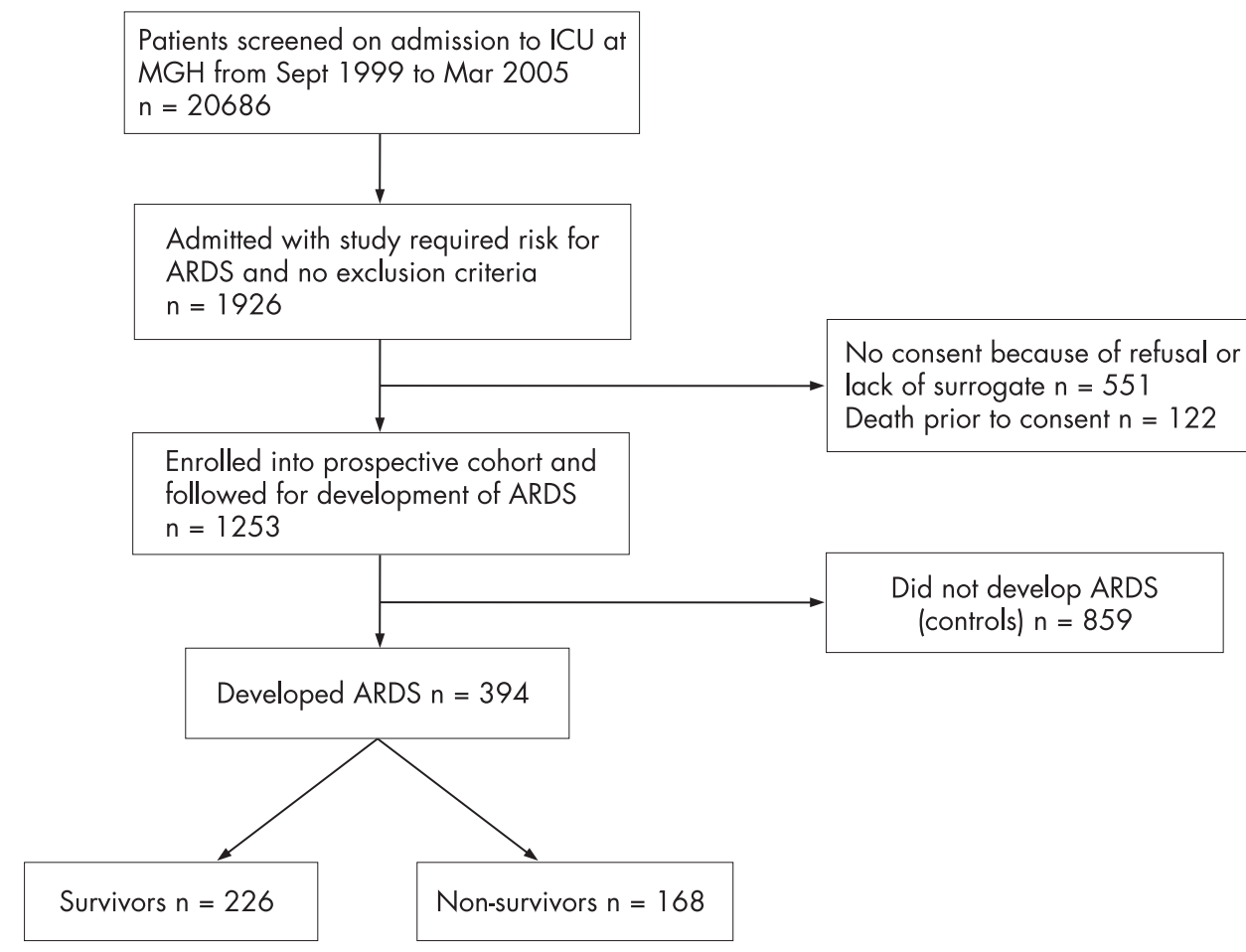

Figure 1 Flow diagram of study design and patient selection for the case-control study. ICU, intensive care unit; MGH, Massachusetts General Hospital, Boston, Massachusetts, USA. Patients who had a previous history of acute respiratory distress syndrome (ARDS) and those who had previously been admitted to the ICU were excluded from being controls. The subjects were all Caucasians. Non-Caucasians were also enrolled but they were excluded from the analysis because they accounted for only $4 \%$ of all enrolment.

\section{DNA isolation and genotyping assays}

Genome DNA was extracted from peripheral blood samples using the Puregene DNA Isolation Kit (Gentra Systems, Minneapolis, Minnesota, USA) following the manufacturer's protocol. The allelic discrimination of the $V E G F$ gene was assessed with the ABI PRISM 7900 Sequence Detection System (Applied Biosystems, Foster City, California, USA) using the fluorogenic 5' nuclease assay with Taqman Minor Groove Binder (MGB) probes. The wide-type Taqman MGB probes were FAM-labelled and the mutant probes were VIC-labelled. The primers and probes for the $-460 C / T$, $+405 C / G$ and the +936C/T polymorphism assays were ordered from Applied Biosystems. Genotyping was performed by laboratory personnel blinded to subject status, and a random $10 \%$ of the samples were repeated to validate genotyping procedures. Two authors reviewed independently all genotyping results.

\section{Analysis of VEGF levels in plasma}

Plasma samples from day 2 after development of ARDS were available from 71 patients for the measurement of circulating
VEGF levels. Blood samples were collected in $10 \mathrm{ml}$ vacuum tubes and centrifuged for $10 \mathrm{~min}$. Plasma samples were stored at $-80^{\circ} \mathrm{C}$ until analysis. Plasma VEGF levels were quantified in duplicate according to the manufacturer's recommendations with a commercially available ELISA kit (R\&D Systems, Minnesota, USA). No statistically significant differences were observed between patients with plasma samples and those without plasma samples in terms of age, sex, APACHE III and risk factors for ARDS.

\section{Statistical analysis}

The demographic variables between different groups were compared by $\chi^{2}$ tests for categorical variables and by the Student $t$ test and/or non-parametric test for continuous variables. The Hardy-Weinberg equilibrium was evaluated using the $\chi^{2}$ test. A logistic regression model was used to assess the effect of $V E G F$ polymorphisms on the mortality of patients with ARDS, with adjustments for potential confounding factors such as age, sex, APACHE III score, diabetes, history of steroid use, number of units of red cells transfused and

Table 1 Characteristics of survivors and non-survivors among patients with ARDS

\begin{tabular}{lllr}
\hline Covariables & Non-survivors $(\mathbf{n = 1 6 8 )}$ & Survivors (n=226) & p Value \\
\hline Mean (SD) age (years) & $67.98(14.75)$ & $53.88(18.62)$ & $<0.01$ \\
Female, $\mathrm{n}(\%)$ & $72(42)$ & $92(41)$ & 0.67 \\
Mean (SD) APACHE III score & $89.60(22.28)$ & $68.95(21.15)$ & $<0.01$ \\
Diabetes, $\mathrm{n}(\%)$ & $31(18)$ & $38(17)$ & 0.70 \\
Chronic liver disease, $\mathrm{n}(\%)$ & $8(10)$ & $6(3)$ & $<0.01$ \\
History of steroid use, $\mathrm{n}(\%)$ & $27(16)$ & $19(8)$ & 0.02 \\
Bilirubin $>2.0 \mathrm{mg} / \mathrm{l}, \mathrm{n}(\%)$ & $41(27)$ & $27(14)$ & $<0.01$ \\
Creatinine $>2.0 \mathrm{mg} / \mathrm{l}, \mathrm{n}(\%)$ & $63(38)$ & $54(24)$ & 0.10 \\
Haematological failure & $48(29)$ & $35(16)$ & $<0.01$ \\
( $\leqslant 80$ 000 platelets $\left./ \mathrm{mm}^{3}\right), \mathrm{n}(\%)$ & & &
\end{tabular}


chronic liver disease. Linkage disequilibrium between the SNPs, haplotypes and their frequencies was estimated using the expectation maximisation algorithm. Association between haplotypes and the risk of ARDS was assessed using an "expectation substitution" approach to account for unknown phase. $^{21}$ Haplotypes were coded as an additive fashion. Mortality from ARDS was regressed on haplotype counts by logistic regression, using the most common haplotype as the reference haplotype. The multiple Cox regression model was applied to test the effect of the VEGF polymorphisms on overall survival, adjusting for confounding factors such as age, sex, APACHE III score, diabetes, history of steroid use and chronic liver disease. The correlations between haplotype probabilities and plasma VEGF levels were estimated by the Spearman correlation test. All statistical analyses were performed using the SAS statistical software package Version 9.1 (SAS Inc, Cary, North Carolina, USA).

\section{RESULTS}

\section{Patient population}

Between September 1999 and March 2005, 1253 Caucasian patients with risk factors of ARDS were enrolled into the prospective cohort. In this study population, 394 (31.4\%) were diagnosed as having ARDS and 859 did not develop ARDS (fig l).

Clinical risk factors for ARDS and baseline characteristics between subjects with and without ARDS are shown in tables 1 and 2 in the online data supplement (available at http:// thorax.bmj.com/supplemental). Among the 394 patients with ARDS studied, the 60 day mortality was $42.6 \%$ (168/394). Logistic analysis showed that older age, APACHE III, steroid use, haematological failure and liver disease were the major prognostic factors for survival (table 1).

\section{VEGF genotypes in relation to ARDS mortality}

Genotyping of the $-460 C / T,+405 C / G$ and $+936 C / T$ polymorphisms was successfully achieved for all subjects and followed the Hardy-Weinberg equilibrium. The genotype frequencies of these three polymorphisms in the current study were broadly similar to those reported by others in Caucasian populations. ${ }^{22-28}$ When overall cases of ARDS were compared with patients without ARDS, no significant difference was found in the distribution of genotypes for any polymorphisms studied ( $p>0.05)$.

We tested for an association between mortality from ARDS and any of the three SNPs among the 394 patients with ARDS (table 2). No significant association was found between the $-460 \mathrm{C} / \mathrm{T}$ or $+405 \mathrm{C} / \mathrm{G}$ polymorphisms and the risk of mortality in patients with ARDS $(\mathrm{p}>0.05)$, but the +936TT genotype was significantly associated with a higher mortality from ARDS than the +936CC genotype (adjusted OR 4.29, 95\% CI 1.12 to $16.40, \mathrm{p}=0.01)$. A similar association was also observed for the +936CT genotype, but this association was not statistically significant (adjusted OR 1.60, 95\% CI 0.90 to 2.80, $\mathrm{p}=0.10$ ). When the mutant +936TT and +936CT were combined to form a united genotype, this combined +936CT+TT genotype was significantly associated with increased mortality compared with the +936CC genotype (adjusted OR 1.98, 95\% CI 1.14 to 3.42, $\mathrm{p}=0.01)$. No significant interactions between any genotypes and age, sex or types of injury were detected.

\section{Association between VEGF haplotypes and ARDS mortality}

Haplotype analyses were conducted to evaluate the combined effect of the three polymorphisms on survival in patients with ARDS. Consistent with observations from early studies, ${ }^{29}$ VEGF $-460 C / T$ and $+405 C / G$ polymorphisms in our study were in strong linkage disequilibrium (correlation coefficient $(\mathrm{R})=0.68$, Lewontin's $\left.\mathrm{D}^{\prime}\left(\mathrm{D}^{\prime}\right)=0.99\right)$, whereas their linkage with the $+936 C / T$ polymorphism was much weaker $(R=0.04$, $\mathrm{D}^{\prime}=0.1$ and $\mathrm{R}=0.1, \mathrm{D}^{\prime}=0.20$, respectively). Six haplotypes with frequencies $>1 \%$ were estimated of the eight possible haplotypes (table 3). Interestingly, the $-460 T+405 C+936 T$ (TCT) haplotype was significantly associated with an increased risk of mortality from ARDS (adjusted OR 2.89, 95\% CI 1.30 to 6.43 , $\mathrm{p}=0.009$ ), and the $-460 \mathrm{C} /+405 G /+936 \mathrm{~T}$ (CGT) haplotype was marginally significantly associated with a higher risk of mortality from ARDS (adjusted OR 1.90, 95\% CI 0.94 to 3.87, $\mathrm{p}=0.07)$. No associations were found between other haplotypes and mortality from ARDS in this study population.

\section{Relationship between VEGF polymorphisms and plasma VEGF levels}

To investigate the relationship between VEGF polymorphisms and circulating VEGF levels, plasma VEGF levels were categorised according to VEGF polymorphisms. Since the plasma levels of VEGF were not normally distributed (Kolmogorov-Smirnov test, $\mathrm{p}=0.01$ ), a non-parametric median two-sample test was used to compare the VEGF levels between different genotype carriers. The median (interquartile range) plasma VEGF level in individuals carrying the $+936 C T+T T$ genotype was significantly lower than that in individuals with the $V E G F+936 C C$ genotype (49 (16-98) pg/ml vs 112 (47162) $\mathrm{pg} / \mathrm{ml}, \mathrm{p}=0.02)$. Based on the sample sizes and median plasma VEGF levels between the +936CT+TT and the $V E G F+936 C C$ carriers, the power to detect a difference at $\alpha=0.05$ level was calculated to be $99 \%$. At haplotype levels, the

Table 2 Associations of VEGF genotypes and mortality from ARDS in all patients $(n=394)$

\begin{tabular}{llll}
\hline Genotypes & $\begin{array}{l}\text { Genotype } \\
\text { frequency }(\%)\end{array}$ & OR* $^{*}(95 \% \mathrm{Cl})$ & p Value* \\
\hline $460 T$ & 29.4 & 1.00 & \\
$460 C T$ & 46.7 & $1.33(0.83$ to 2.14$)$ & 0.24 \\
$460 C C$ & 23.9 & $0.77(0.44$ to 1.35$)$ & 0.37 \\
$405 G G$ & 43.9 & 1.00 & 0.20 \\
$405 C G$ & 43.4 & $1.37(0.84$ to 2.24$)$ & 0.69 \\
$405 C C$ & 12.7 & $0.87(0.43$ to 1.74$)$ & 0.10 \\
$936 C C$ & 73.9 & 1.00 & 0.03 \\
$936 C T$ & 22.8 & $1.60(0.90$ to 2.80$)$ & 0.01 \\
$936 T \pi$ & 3.3 & $4.29(1.12$ to 16.40$)$ & 0.005 \\
$936 C T+\pi$ & 26.1 & $1.98(1.14$ to 3.42$)$ & \\
Prrend $t$ & $1.93(1.10-1.94)$ & & \\
\hline
\end{tabular}

*Estimated by logistic regression models, adjusting for age, sex, history of alcohol abuse, diabetes, steroid use, APACHE III score and multiple (>1) risk factors for ARDS.

†The genotypes were coded as continuous variables 1,2 and 3 for wild type, heterozygous and homozygous genotypes, respectively) and entered together into the logistic regression model. 
Table 3 Associations between VEGF haplotypes and ARDS mortality

\begin{tabular}{llll}
\hline Haplotypest & Haplotype frequency $(\%)$ & OR $^{*}(95 \% \mathrm{Cl})$ & $\mathbf{p ~ V a l u e ^ { * }}$ \\
\hline Global test & 39.6 & 1.0 & 0.026 \\
CGC & 28.9 & $0.85(0.59$ to 1.24$)$ & 0.4 \\
TCC & 16.6 & $0.95(0.58$ to 1.55$)$ & 0.8 \\
TGC & 7.4 & $1.90(0.94$ to 3.83$)$ & 0.07 \\
CGT & 5.3 & $2.89(1.30$ to 6.43$)$ & 0.009 \\
TCT & 1.7 & $1.09(0.20$ to 5.84$)$ & 0.92 \\
TGT & &
\end{tabular}

*Estimated by logistic regression models, adjusting for age, sex, history of alcohol abuse, diabetes, steroid use, APACHE III and multiple risk of (>1) of ARDS.

tThe order of the polymorphisms is as follows: $-460 \mathrm{C} / \mathrm{T},+405 \mathrm{C} / \mathrm{G},+936 \mathrm{C} / \mathrm{T}$.

plasma VEGF levels were inversely correlated with haplotype CGT probability (Spearman coefficient $-0.26, \mathrm{p}<0.05$ ). A similar correlation was also observed between plasma VEGF levels and the haplotype TCT probability, but this correlation did not reach statistical significance (coefficient -0.14 , $\mathrm{p}=0.26$ ). Thus, haplotypes containing the $936 \mathrm{~T}$ allele tended to correlate with lower plasma VEGF levels. There was no significant difference between survivors and non-survivors in plasma levels of VEGF $(\mathrm{p}>0.05)$.

\section{Association between VEGF polymorphisms and ARDS survival}

The Cox proportional hazard model was used to analyse the associations between $V E G F$ polymorphisms and survival in patients with ARDS. After adjusting for other predictors of survival (age, sex, APACHE III score, diabetes, history of steroid use, haematological failure and chronic liver disease), both the $+936 C T+T T$ genotype (hazard ratio (HR) 1.71 , 95\% CI 1.09 to $2.72, \mathrm{p}=0.02$ ) and haplotype TCT (HR 2.11, 95\% CI 1.32 to 3.37 , $\mathrm{p}<0.01$ ) were significantly associated with lower survival probability, consistent with the results assessed by logistic regression models.

\section{DISCUSSION}

Our data have shown that the +936TT and +936CT+TT genotypes of the $V E G F$ gene are significantly associated with an increased risk of mortality from ARDS. At the haplotype level, the TCT haplotype was significantly associated with a higher mortality from ARDS. In addition, we showed that VEGF polymorphisms contributing to increased mortality in patients with ARDS were correlated with lower plasma levels of VEGF.

This study has a number of strengths. First, the prospective determination of ARDS using the AECC definition minimised phenotype misclassification since there is no diagnostic gold standard for ARDS. Second, the VEGF polymorphisms and the circulating VEGF levels were determined in a parallel manner. Thus, the functional effect of VEGF polymorphisms can be assessed. Third, restricting analyses to Caucasian patients reduced the possibility of altered genotype frequencies in different ethnicities.

Among the three functional SNPs studied, none of the $-460 \mathrm{C} /$ $T$ and $+405 C / G$ polymorphisms was found to have a significant effect on mortality from ARDS. However, the +936TT and the combined+936CT+TT genotypes were significantly associated with ARDS mortality, suggesting that +936 polymorphisms may have a stronger role than either the $-460 C / T$ or $+405 C / G$ polymorphisms in VEGF gene functions. Recently, a small case-control study found that the frequency of the combined $+936 C T+T T$ genotype in patients with ARDS was significantly higher than in controls. This study also found that the +936CT and TT genotypes were associated with the APACHE III score in patients with ARDS, but the association between the +936 CT/ TT genotype and increased mortality from ARDS did not reach statistical significance $(\mathrm{OR}>1.80, \mathrm{p}>0.05) .{ }^{26}$ In the present larger study, a significant association between the $+936 C T+T T$ genotype and mortality was found in patients with ARDS. At the haplotype level, the haplotype containing the +936T allele was also associated with mortality from ARDS, further supporting the effects of $+936 \mathrm{C} / \mathrm{T}$ variants on outcomes in patients with ARDS.

Our data showed that the +936CT/TT genotype was significantly correlated with lower plasma levels of VEGF, consistent with the functions of this polymorphism reported in other studies. ${ }^{14}{ }^{15}$ Although the plasma VEGF levels were measured at only one time point and may not therefore fully reflect the temporal response of VEGF over the course of the disease, our results suggest that VEGF may be a protective factor in the severity of ARDS. Decreased levels of VEGF in the lungs have been correlated with a higher lung injury score and lower survival rates in patients with ARDS, ${ }^{10}{ }^{11}{ }^{30}$ whereas increased VEGF production in the lungs resulted in less lung injury. ${ }^{31}$ In animal models, administration of VEGF prevented lung injuryinduced death. ${ }^{30}$ In alveolar epithelial cells, VEGF mRNA expression increased during recovery from oxygen injury. ${ }^{33}$ In neonatal rats exposed to high oxygen levels, intraocular injection of VEGF at the onset of experimental hyperoxia prevented apoptotic death of endothelial cells and rescued the retinal vasculature. ${ }^{34}$ Taken together, these findings indicate that VEGF may function as a protective factor in ARDS.

Although the haplotypes predicted in the present study were identical to those previously reported, the overall distribution of haplotype frequencies in the present study was different from that in Chinese and Korean populations. ${ }^{29}{ }^{35}$ Different haplotype frequencies in different populations may be due to different ethnic backgrounds or different disease states. Interestingly, the frequencies of TCT (5.3\%) and CGT (7.4\%) haplotypes in the present study were similar to those reported in the Chinese population $\left(6.0 \%\right.$ and $6.4 \%$, respectively). ${ }^{29}$ In Asian populations, both the TCT and CGT haplotypes were significantly implicated in a reduced risk of lung cancer, and the CGT haplotype was associated with decreased survival in patients with breast cancer. ${ }^{29}$ These observations suggested that TCT and CGT haplotypes may play an important role in disease processes. However, since these previous studies did not measure circulating VEGF levels, the mechanisms connecting the VEGF haplotypes with development or severity of the disease are unclear. Our study not only indicated that the TCT haplotype was associated with mortality from ARDS, but also showed that the CGT and TCT haplotypes correlated with lower circulating levels of VEGF protein, which suggests that these functional haplotypes may contribute to mortality from ARDS by affecting VEGF production.

We acknowledge some potential limitations to this study. First, the sample size was relatively small. Additional studies with larger populations are required to confirm the associations between the VEGF haplotypes and mortality in patients with 
ARDS. Second, the VEGF gene is a polymorphic gene, and the present study only investigated three SNPs. Further studies on other functional VEGF SNPs are needed to define the role of the $V E G F$ polymorphisms in prognosis in ARDS. Third, measurement of plasma VEGF levels was done at one time point so the role of the plasma VEGF level in the natural history of ARDS remains to be defined. Finally, since genetic polymorphisms often vary between ethnic groups, further studies are necessary to clarify the association between the VEGF polymorphisms and the outcome in patients with ARDS in diverse ethnic populations.

In summary, the findings of this study suggest that the +936TT and +936CT+TT genotypes and the TCT haplotype of the $V E G F$ gene contribute to increased mortality and to interindividual variations in plasma VEGF levels in patients with ARDS. The associations found in this study are biologically plausible and may have significant implications for the treatment of ARDS.

\section{ACKNOWLEDGEMENTS}

The authors thank Weiling Zhang, Kelly McCoy, Thomas McCabe, Marcia Chertok and Julia Shin for patient recruitment; Andrea Shafer and Lia Shimada for research support; Ian James for laboratory expertise; and Janna Frelich and Lucille Pothier for data management.

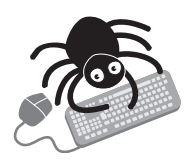

Clinical risk factors for ARDS and baseline characteristics between subjects with and without ARDS are shown in tables 1 and 2 in the online data supplement available at http://thorax.bmi.com/supplemental.

\section{Authors' affiliations}

R Zhai, W Zhou, L Su, D C Christiani, Department of Environmental Health, Harvard School of Public Health, Boston, Massachusetts, USA

M N Gong, Division of Pulmonary and Critical Care Medicine, Mount Sinai School of Medicine, New York, USA

T B Thompson, D C Christiani, Pulmonary and Critical Care Unit, Department of Medicine, Massachusetts General Hospital, Harvard Medical School, Boston, Massachusetts, USA

P Kraft, Department of Biostatistics, Harvard School of Public Health, Boston, Massachusetts, USA

This work was supported by grants from National Institute of Health (HL60710, ESO0002, and K23 HL67197).

Competing interests: Dr Christiani is a paid scientific advisor to Gentra Corporation.

\section{REFERENCES}

1 Matthay MA, Zimmerman GA, Esmon C, et al. Future research directions in acute lung injury: summary of a National Heart, Lung, and Blood Institute Working Group. Am J Respir Criti Care Med 2003:167:1027-35.

2 Piantadosi CA, Schwartz DA. The acute respiratory distress syndrome. Ann Intern Med 2004;141:460-70.

3 Berthiaume Y, Lesur O, Dsgenais A. Treatment of adult respiratory distress syndrome: plea for rescue therapy of the alveolar epithelium. Thorax 1999;54:50-60.

4 Bernard GR, Artigas A, Brigham KL, et al. The American-European Consensus Conference on ARDS. Definition, mechanisms, relevant outcomes, and clinical trial coordination. Am J Respir Crit Care Med 1994;149:818-24.

5 Ware LB, Conner ER, Matthay MA. von Willebrand factor antigen is an independent marker of poor outcome in patients with early acute lung injury. Crit Care Med 2001;29:2325-31.

6 Mura M, dos Santos CC, Stewart D, et al. Vascular endothelial growth factor and related molecules in acute lung injury. J Appl Physiol 2004;97:1605-17.
7 Boussat S, Eddahibi S, Coste A, et al. Expression and regulation of vascular endothelial growth factor in human pulmonary epithelial cells. Am J Physiol Lung Cell Mol Physiol 2000;279:L371-8.

8 Kaner RJ, Ladetto JV, Singh R, et al. Lung overexpression of the vascular endothelial growth factor gene induces pulmonary edema. Am J Respir Cell Mol Biol 2000;22:657-64.

9 Ferrara N. Vascular endothelial growth factor: basic science and clinical progress. Endocr Rev 2004;25:581-11.

10 Thickett DR, Armstrong L, Millar AB. A role for vascular endothelial growth factor in acute and resolving lung injury. Am J Respir Criti Care Med 2002;166:1332-7.

11 Abadie Y, Bregeon F, Papazian L, et al. Decreased VEGF concentration in lung tissues and vascular injury during ARDS. Eur Respir J 2005;25:139-46.

12 Hanaoka M, Dromam Y, Naramoto A, et al. Vascular endothelial growth factor in patients with high-altitude pulmonary edema. J Appl Physiol 2003;94:1836-40.

13 Maniscalco WM, Watkins RH, Finkelstein JN, et al. Vascular endothelial growth factor mRNA increases in alveolar epithelial cells during recovery from oxygen injury. Am J Respir Cell Mol Biol 1995;13:377-86.

14 Renner W, Kotschan C, Hoffman B, et al. A common 936C/T mutation in the gene for vascular endothelial growth factor is associated with vascular endothelial growth factor plasma levels. J Vasc Res 2000;37:443-8.

15 Sciacca FL, Ciusani E, Silvani A, et al. Genetic and plasma markers of venous thromboembolism in patients with high grade glioma. Clin Cancer Res 2004;10:1312-7.

16 Watson CJ, Webb NJA, Bottomley MJ, et al. Identification of polymorphisms within the vascular endothelial growth factor (VEGF) gene: correlation with variation in VEGF protein production. Cytokine 2000;12:1232-5.

17 Han SW, Kim GW, Soe JS, et al. VEGF gene polymorphisms and susceptibility to rheumatoid arthritis. Rheumatology 2004;43:1173-7.

18 Shahbazi M, Fryer AA, Pravica V, et al. Vascular endothelial growth factor gene polymorphisms are associated with acute renal allograft rejection. J Am Soc Nephrol 2002; 13:260-4.

19 Gong MN, Zhou W, Xu LL, Miller D, et al. Polymorphism in the surfactant protein$B$ gene, gender, and the risk of direct pulmonary injury and ARDS. Chest 2004; 125:203-11.

20 Gong MN, Zhou W, Williams P, et al. -380GA and TNFB polymorphisms in acute respiratory distress syndrome. Eur Respir J 2005;26:382-9.

21 Kraft P, Cox DG, Paynter RA, et al. Accounting for haplotype uncertainty in matched association studies: a comparison of simple and flexible techniques. Genet Epidemiol 2005;28:261-72.

22 Sakao SK, Tatsumi T, Hashimato $\mathrm{H}$, et al. Vascular endothelial growth factor and the risk of smoking-related COPD. Chest 2003;124:323-7.

23 Marohashi K, Takada T, Omori K, et al. Vascular endothelial growth factor gene polymorphisms in Japanese patients with sarcoidosis. Chest 2003;123:1520-6.

24 Awata T, Inoue K, Kurihara S, et al. A common polymorphism in the 5' untranslated region of the VEGF gene is associated with diabetic retinopathy in type 2 diabetes. Diabetes 2002;51:1630-5.

25 Stevens A, Soden J, Brenchley PE, et al. Haplotype analysis of the polymorphic human vascular endothelial growth factor gene promoter. Cancer Res 2003;63:812-6.

26 Medford ARL, Keen $\sqcup$, Bidwell JL, et al. Vascular endothelial growth factor gene polymorphism and acute respiratory distress syndrome. Thorax 2005;60:244-8.

27 Krippl P, Langsenlehner U, Renner W, et al. A common $936 \mathrm{C} / \mathrm{T}$ gene polymorphism of vascular endothelial growth factor is associated with decreased breast cancer risk. Int J Cancer 2003;106:468-71.

28 Cancer Genome Anatomy Project. SNP500 cancer database, http:// snp500cancer.nci.nih.gov/home_1.cfm.

29 Lu $\mathrm{H}$, Shun $\mathrm{XO}, \mathrm{Cui} Y$, et al. Association of genetic polymorphisms in the VEGF gene with breast cancer survival. Cancer Res 2005;65:5015-9.

30 Maitre B, Boussat S, Jean D, et al. Vascular endothelial growth factor synthesis in the acute phase of experimental and clinical lung injury. Eur Respir J $2001 ; 18: 100-6$.

31 Corne J, Chupp G, Lee CG, et al. IL-13 stimulates vascular endothelial cell growth factor and protects against hyperoxic acute lung injury. J Clin Invest 2000;106:783-91.

32 Compernolle V, Brusselmans K, Acker T, et al. Loss of HIF-2 alpha and inhibition of VEGF impair foetal lung maturation, whereas treatment with VEGF prevents fatal respiratory distress in premature mice. Nat Med 2002;8:702-10.

33 Maniscalco WM, Watkins RH, Finkelstein JN, et al. Vascular endothelial growth factor mRNA increases in alveolar epithelial cells during recovery from oxygen injury. Am J Respir Cell Mol Biol 1995;13:377-86.

34 Alon T, Hemo I, Itin A, et al. Vascular endothelial growth factor acts as a survival factor for newly formed retinal vessels and has implications for retinopathy of prematurity. Nat Med 1995;10:1024-8.

35 Lee SJ, Lee SY, Jeon HS, et al. Vascular endothelial growth factor gene polymorphisms and risk of primary lung cancer. Cancer Epidemiol Biomarker Preven 2005; 14:571-5. 